\title{
Mean-value properties of real analytic functions
}

\author{
GrZEgORZ EYSIK
}

\begin{abstract}
We extend the Pizzetti formulas, i.e., expansions of the solid and spherical means of a function in terms of the radius of the ball or sphere, to the case of real analytic functions and to functions of Laplacian growth. We also give characterizations of these functions. As an application we give a characterization of solutions analytic in time of the initial value problem for the heat equation $\partial_{t} u=\Delta u$ in terms of holomorphic properties of the solid and/or spherical means of the initial data.
\end{abstract}

Mathematics Subject Classification (2010). 31B30, 30D10, 35K05.

Keywords. Mean-values, Polyharmonic functions,

Real analytic functions, Pizzetti's formula, Heat equation.

1. Introduction. The mean-value property of harmonic functions, i.e., solutions of the Laplace equation $\Delta u=0$, where $\Delta=\sum_{i=1}^{d} \frac{\partial^{2}}{\partial x_{i}^{2}}$, is well known. Namely, if $u$ is harmonic on a domain $\Omega \subset \mathbb{R}^{d}$, then for every closed ball $B(\stackrel{\circ}{x}, R) \subset \Omega$ with center $\stackrel{\circ}{x} \in \Omega$ and radius $R>0$ the average of $u$ over $B(\stackrel{\circ}{x}, R)$, and over the sphere $S(\stackrel{\circ}{x}, R)$, equals $u(\stackrel{\circ}{x})$, i.e.,

$$
\begin{aligned}
& u(\grave{x})=\frac{1}{\sigma(d) R^{d}} \int_{B(\grave{x}, R)} u(x) d x, \\
& u(\grave{x})=\frac{1}{d \sigma(d) R^{d-1}} \int_{S(\grave{x}, R)} u(x) d S(x),
\end{aligned}
$$

where $\sigma(d)=\pi^{d / 2} / \Gamma(d / 2+1)$ (with $\Gamma$ the Euler $\Gamma$-function) is the volume of the unit ball in $\mathbb{R}^{d}$ and $d S$ denotes the surface measure on $S(\stackrel{\circ}{x}, R)$. Conversely, if a continuous function $u$ satisfies (1.1a) for every ball (or (1.1b) for every sphere) in $\Omega$, then $u$ is twice continuously differentiable and harmonic on $\Omega$. A generalization of the mean-value property and its converse to polyharmonic functions, i.e., solutions of $\Delta^{m} u, m \in \mathbb{N}$, was studied by many authors. It turns 
out that in this case the spherical and solid means can be expressed by some polynomials in the radius of the ball or sphere. In the case of spherical means in dimensions $d=2,3$ the mean-value formula was established already in 1909 by Pizzetti $[14,15]$. The converse to the mean-value property for polyharmonic functions in dimension $d=2$ was first proved by Sbrana [17]. Extensions of the Pizzetti mean-value formula and the converse mean-value property for polyharmonic functions to the case of spherical and solid means in arbitrary dimension were obtained by Nicolesco [13]; their simple proofs based on differential relations between spherical and solid means can be found in [10]. Some other theorems on mean-value properties for polyharmonic and real analytic functions have been obtained by Ghermanesco [7,8], Friedman [6], Bramble and Payne [4], Sampson [16], Bojanov [3], Zalcman [18], and others.

In this paper we extend the mean-value formulas to the case of real analytic functions and obtain a characterization of these functions in terms of integral means over balls or spheres. We also obtain a similar characterization of functions of Laplacian growth. As an application we study the problem of analyticity in time of solutions to the initial value problem for the heat equation $\partial_{t} u=\Delta u$ with real analytic initial data $u(0, \cdot)=u_{0}$. We prove that the solution $u$ is analytic in time at $t=0$ if and only if the integral means of $u_{0}$ over balls or spheres of radius $R$ can be extended to entire functions of $R$ of exponential order at most 2.

2. Solid and spherical means. Throughout the paper $\Omega$ denotes a domain, i.e., a connected, open set in $\mathbb{R}^{d}$. Let $u$ be a continuous function defined on $\Omega$. For any $\stackrel{\circ}{x} \in \Omega$ and $0<R<\operatorname{dist}(\stackrel{\circ}{x}, \partial \Omega)$ we denote by $M(u, \stackrel{\circ}{x} ; R)$ and $N(u, \stackrel{\circ}{x} ; R)$ the integral means of $u$ over the closed ball $B(\stackrel{\circ}{x}, R) \subset \Omega$ and the sphere $S(\stackrel{\circ}{x}, R) \subset \Omega$ respectively, i.e.,

$$
\begin{array}{r}
M(u, \stackrel{\circ}{x} ; R)=\frac{1}{\sigma(d) R^{d}} \int_{B(\stackrel{\circ}{x}, R)} u(x) d x, \\
N(u, \stackrel{\circ}{x}, R)=\frac{1}{d \sigma(d) R^{d-1}} \int_{S(\stackrel{\circ}{x}, R)} u(x) d S(x) .
\end{array}
$$

The relations between $M(u, \stackrel{\circ}{x} ; R)$ and $N(u, \stackrel{\circ}{x} ; R)$ are given in the following result.

Lemma 2.1. [10, Lemma 1]. Let $u$ be a continuous function on $\Omega \subset \mathbb{R}^{d}$. Then for any $\stackrel{\circ}{x} \in \Omega$ and $0<R<\operatorname{dist}(\stackrel{\circ}{x}, \partial \Omega)$ we have

$$
\left(\frac{R}{d} \frac{\partial}{\partial R}+1\right) M(u, \stackrel{\circ}{x} ; R)=N(u, \stackrel{\circ}{x} ; R) .
$$

If we further assume that $u$ has continuous partial derivatives up to second order, then

$$
\frac{d}{R} \frac{\partial}{\partial R} N(u, \stackrel{\circ}{x} ; R)=M(\Delta u, \stackrel{\circ}{x} ; R) .
$$

The proof of (2.1) follows from the computation of $M(u, \stackrel{\circ}{x} ; R)$ in spherical coordinates while that of (2.2) by using the Green formula. For details see [10]. 
3. Mean-value properties for real analytic functions. In this section we prove mean-value formulas for solid and spherical means of real analytic functions. Conversely, real analytic functions are characterized in terms of solid or spherical means.

Denote by $\mathcal{A}(\Omega)$ the set of real analytic functions on $\Omega \subset \mathbb{R}^{d}$, i.e., $u \in \mathcal{A}(\Omega)$ if for any $\stackrel{\circ}{x} \in \Omega$ one can find $\rho>0$ such that

$$
u(x)=\sum_{\ell \in \mathbb{N}_{0}^{d}} \frac{1}{\ell_{1} ! \cdots \ell_{d} !} \frac{\partial^{|\ell|}}{\partial x^{\ell}} u(\stackrel{\circ}{x})(x-\stackrel{\circ}{x})^{\ell} \quad \text { for }\|x-\stackrel{\circ}{x}\|<\rho,
$$

where $\|\cdot\|$ denotes the Euclidean norm, $\mathbb{N}_{0}=\mathbb{N} \cup\{0\},|\ell|=\ell_{1}+\cdots+\ell_{d}$ and $x^{\ell}=x_{1}^{\ell_{1}} \cdots x_{d}^{\ell_{d}}$.

Theorem 3.1. (Mean-value property). Let $u \in \mathcal{A}(\Omega)$ and $\stackrel{\circ}{x} \Omega$. Then $M(u, \stackrel{\circ}{x} ; R)$ and $N(u, \stackrel{\circ}{x} ; R)$ are real analytic functions at the origin and for $R$ small enough

$$
\begin{aligned}
& M(u, \stackrel{\circ}{x} ; R)=\sum_{k=0}^{\infty} \frac{\Delta^{k} u(\stackrel{\circ}{x})}{4^{k}\left(\frac{d}{2}+1\right)_{k} k !} R^{2 k}, \\
& N(u, \stackrel{\circ}{x} ; R)=\sum_{k=0}^{\infty} \frac{\Delta^{k} u(\stackrel{\circ}{x})}{4^{k}\left(\frac{d}{2}\right)_{k} k !} R^{2 k},
\end{aligned}
$$

where for $a \in \mathbb{R},(a)_{0}=1$ and $(a)_{k}=a(a+1) \cdots(a+k-1)$ for $k \in \mathbb{N}$.

Proof. For simplicity assume that $\stackrel{\circ}{x}=0$ and (3.1) holds for $x \in B(0, \rho)$. Set $B(R)=B(0, R)$ with $R<\rho$. Note that if at least one of the exponents $\ell_{1}, \ldots, \ell_{d}$ is odd, then the integral of $x^{\ell}=x_{1}^{\ell_{1}} \cdots x_{d}^{\ell_{d}}$ over $B(R)$ vanishes. Next using $[5$, formula 676,11$)]$ we derive for $k \in \mathbb{N}_{0}$ and $\kappa \in \mathbb{N}_{0}^{d}$ with $|\kappa|=k$,

$$
\begin{aligned}
\frac{1}{\sigma(d) R^{d}} \int_{B(R)} x_{1}^{2 \kappa_{1}} \cdots x_{d}^{2 \kappa_{d}} d x & =\frac{R^{2 k}}{\sigma(d)} \int_{B(1)} y_{1}^{2 \kappa_{1}} \cdots y_{d}^{2 \kappa_{d}} d y \\
& =\frac{R^{2 k}}{\sigma(d)} \frac{\Gamma\left(\kappa_{1}+\frac{1}{2}\right) \cdots \Gamma\left(\kappa_{d}+\frac{1}{2}\right)}{\Gamma\left(\frac{d}{2}+k+1\right)} \\
& =\frac{\left(\frac{1}{2}\right)_{\kappa_{1}} \cdots\left(\frac{1}{2}\right)_{\kappa_{d}}}{\left(\frac{d}{2}+1\right)_{k}} R^{2 k},
\end{aligned}
$$

since $\sigma(d)=\pi^{d / 2} / \Gamma(d / 2+1), \Gamma(1 / 2)=\sqrt{\pi}$ and $z \Gamma(z)=\Gamma(z+1)$. Hence

$$
\begin{aligned}
M(u, \stackrel{\circ}{x} ; R) & =\sum_{k=0}^{\infty} \sum_{\kappa \in \mathbb{N}_{0}^{d},|\kappa|=k} \frac{\partial^{2 k} u(\stackrel{\circ}{x})}{\partial x^{2 \kappa}} \frac{1}{\left(2 \kappa_{1}\right) ! \cdots\left(2 \kappa_{d}\right) !} \frac{\left(\frac{1}{2}\right)_{\kappa_{1}} \cdots\left(\frac{1}{2}\right)_{\kappa_{d}}}{\left(\frac{d}{2}+1\right)_{k}} R^{2 k} \\
& =\sum_{k=0}^{\infty} \frac{R^{2 k}}{4^{k}\left(\frac{d}{2}+1\right)_{k} k !} \sum_{\kappa \in \mathbb{N}_{0}^{d},|\kappa|=k} \frac{k !}{\kappa_{1} ! \cdots \kappa_{d} !} \frac{\partial^{2 k} u(\stackrel{\circ}{x})}{\partial x^{2 \kappa}} \\
& =\sum_{k=0}^{\infty} \frac{\Delta^{k} u(\stackrel{\circ}{x})}{4^{k}\left(\frac{d}{2}+1\right)_{k} k !} R^{2 k} .
\end{aligned}
$$


To prove the convergence of the series (3.4) note that by the Cauchy inequalities for any $\bar{R}=\left(\bar{R}_{1}, \ldots, \bar{R}_{d}\right) \in \mathbb{R}_{+}^{d}$ with $\|\bar{R}\|<\rho$ one can find $C<\infty$ depending only on $\|\bar{R}\|$ such that for any $\ell \in \mathbb{N}_{0}^{d}$,

$$
\left|\frac{\partial^{|\ell|} u(\stackrel{x}{)}}{\partial x^{\ell}}\right| \leq \frac{C}{\bar{R}_{1}^{\ell_{1}} \cdots \bar{R}_{d}^{\ell_{d}}} \ell_{1} ! \cdots \ell_{d} !
$$

Now choosing $\bar{R}_{i}=\|\bar{R}\| / \sqrt{d}$ for $i=1, \ldots, d$ we get

$$
\left|\frac{\partial^{|\ell|} u(\stackrel{x}{\partial})}{\partial x^{\ell}}\right| \leq C\left(\frac{\sqrt{d}}{\|\bar{R}\|}\right)^{|\ell|} \quad \ell_{1} ! \cdots \ell_{d} ! \quad \text { for } \ell \in \mathbb{N}_{0}^{d} .
$$

Next observe that

$$
\begin{aligned}
\sum_{\kappa \in \mathbb{N}_{0}^{d},|\kappa|=k}\left(\frac{1}{2}\right)_{\kappa_{1}} \cdots\left(\frac{1}{2}\right)_{\kappa_{d}} & \leq \sum_{\kappa \in \mathbb{N}_{0}^{d},|\kappa|=k} \kappa_{1} ! \cdots \kappa_{d} ! \\
& \leq \sum_{\kappa \in \mathbb{N}_{0}^{d},|\kappa|=k} k !=\frac{(k+d-1) !}{(d-1) !}
\end{aligned}
$$

So

$$
\begin{aligned}
\sum_{k=0}^{\infty} \frac{\left|\Delta^{k} u(\stackrel{\circ}{x})\right|}{4^{k}\left(\frac{d}{2}+1\right)_{k} k !} R^{2 k} & \leq \sum_{k=0}^{\infty} \sum_{\kappa \in \mathbb{N}_{0}^{d},|\kappa|=k} C\left(\frac{\sqrt{d}}{\|\bar{R}\|}\right)^{2 k} \frac{\left(\frac{1}{2}\right)_{\kappa_{1}} \cdots\left(\frac{1}{2}\right)_{\kappa_{d}}}{\left(\frac{d}{2}+1\right)_{k}} R^{2 k} \\
& \leq \frac{C}{(d-1) !} \sum_{k=0}^{\infty} \frac{(k+d-1) !}{\left(\frac{d}{2}+1\right)_{k}}\left(\frac{\sqrt{d} R}{\|\bar{R}\|}\right)^{2 k} .
\end{aligned}
$$

Hence the series (3.4) converges for $|R|<\rho / \sqrt{d}$.

Finally, applying (2.1) we get (3.3).

Theorem 3.2. (Converse to the mean-value property). Let $\rho: \Omega \rightarrow \mathbb{R}_{+}$be $a$ continuous positive function and $u \in C^{\infty}(\Omega)$. If the series

$$
\widetilde{M}(x ; R)=\sum_{k=0}^{\infty} \frac{\Delta^{k} u(x)}{4^{k}\left(\frac{d}{2}+1\right)_{k} k !} R^{2 k}
$$

is locally uniformly convergent in $\{(x, R): x \in \Omega,|R|<\rho(x)\}$, then $u \in \mathcal{A}(\Omega)$ and $M(u, x ; R)=\widetilde{M}(x ; R)$ for $x \in \Omega$ and $0<R<\min (\rho(x)$, $\operatorname{dist}(x, \partial \Omega))$.

Proof. Fix a compact set $K \Subset \Omega$, and set $\rho=\inf _{x \in K} \rho(x)>0$. Then the assumption implies that

$$
\frac{\Delta^{k} u(x)}{4^{k}\left(\frac{d}{2}+1\right)_{k} k !} R^{2 k} \rightarrow 0 \quad \text { as } k \rightarrow \infty
$$

uniformly on $K \times\left\{|R| \leq \rho_{1}\right\}$ with any $\rho_{1}<\rho$. So for any $\rho_{1}<\rho$ there exists a constant $C\left(\rho_{1}\right)<\infty$ such that

$$
\sup _{x \in K}\left|\Delta^{k} u(x)\right| \leq C\left(\rho_{1}\right) \cdot 4^{k}(d / 2+1)_{k} k ! \rho_{1}^{-2 k} \quad \text { for } k \in \mathbb{N}_{0} .
$$


Applying the inequalities $(d / 2+1)_{k} \leq(d / 2+1)^{k} k$ ! and $2^{k}(k !)^{2} \leq(2 k)$ !, we see that for any compact set $K \Subset \Omega$ one can find $C<\infty$ and $L<\infty$ such that

$$
\sup _{x \in K}\left|\Delta^{k} u(x)\right| \leq C L^{2 k}(2 k) ! \quad \text { for } k \in \mathbb{N}_{0} .
$$

But by $[1$, Theorem 2.2 in Chapter II] this inequality implies that $u \in \mathcal{A}(\Omega)$, (see also [12, Theorem 3.8.9]). Finally, by Theorem 3.1 we get $\widetilde{M}(x ; R)=$ $M(u, x ; R)$.

Corollary 3.3. Under the assumptions of Theorem 3.2 if the series

$$
\tilde{N}(x ; R)=\sum_{k=0}^{\infty} \frac{\Delta^{k} u(x)}{4^{k}\left(\frac{d}{2}\right)_{k} k !} R^{2 k}
$$

is locally uniformly convergent in $\{(x, R): x \in \Omega,|R|<\rho(x)\}$, then $u \in \mathcal{A}(\Omega)$ and $N(u, x ; R)=\widetilde{N}(x ; R)$ for $x \in \Omega$ and $0<R<\min (\rho(x)$, $\operatorname{dist}(x, \partial \Omega))$.

Corollary 3.4. Let $u \in C^{\infty}(\Omega)$. If $u$ is polyharmonic in $\Omega$, then $u \in \mathcal{A}(\Omega)$.

Proof. Indeed, polyharmonicity of $u$ implies that the sum in (3.5) is finite, so Theorem 3.2 applies.

4. Functions of Laplacian growth. In order to control the growth of iterated Laplacians of smooth functions Aronszajn et al. [1] introduced the notion of Laplacian growth.

Definition 4.1. Let $\varrho>0$ and $\tau \geq 0$. A function $u$ smooth on $\Omega \subset \mathbb{R}^{d}$ is of Laplacian growth $(\varrho, \tau)$ on $\Omega$ if for every compact set $K \Subset \Omega$ and $\varepsilon>0$ one can find $C=C(K, \varepsilon)<\infty$ such that

$$
\sup _{x \in K}\left|\Delta^{k} u(x)\right| \leq C(2 k) !^{1-1 / \varrho}(\tau+\varepsilon)^{2 k} \quad \text { for any } k \in \mathbb{N}_{0} .
$$

Recall also

Definition 4.2. [2]. Let $\varrho>0$ and $\tau \geq 0$. An entire function $F$ is said to be of exponential growth $(\varrho, \tau)$ if for every $\varepsilon>0$ one can find $C_{\varepsilon}$ such that

$$
\sup _{|z| \leq R}|F(z)| \leq C_{\varepsilon} \exp \left\{(\tau+\varepsilon) R^{\varrho}\right\} \quad \text { for any } R<\infty .
$$

The exponential growth of an entire function can be expressed in terms of estimates of its Taylor coefficients.

Theorem 4.3. [2, Theorems 2.2.2, 2.2.10]. Let $\varrho>0, \tau \geq 0$, and let $F(z)=$ $\sum_{n=0}^{\infty} a_{n} z^{n}$ be an entire function. Then $F$ is of exponential growth $(\varrho, \tau)$ if and only if for any $\varepsilon>0$ one can find $C_{\varepsilon}<\infty$ such that

$$
\left|a_{n}\right| \leq C_{\varepsilon}\left(\frac{e \varrho \tau+\varepsilon}{n}\right)^{n / \varrho} \text { for any } n \in \mathbb{N} .
$$

Definition 4.4. Let $\varrho>0$ and $\tau \geq 0$. We say that a function $F$ defined on $\Omega \times \mathbb{C}, \Omega \subset \mathbb{R}^{d}$, is entire of exponential growth $(\varrho, \tau)$ locally uniformly in $\Omega$ if for any compact set $K \Subset \Omega$ and $\varepsilon>0$ one can find $C_{K, \varepsilon}$ such that

$$
\sup _{x \in K} \sup _{|z| \leq R}|F(x, z)| \leq C_{K, \varepsilon} \exp \left\{(\tau+\varepsilon) R^{\varrho}\right\} \quad \text { for any } R<\infty .
$$


It turns out that a function $u$ of Laplacian growth $(\varrho, \tau)$ on $\Omega$ is, in fact, real analytic on $\Omega$ [1, Theorem 2.2 in Chapter II]. So the solid and spherical means $M(u, x ; R)$ and $N(u, x ; R)$ are well defined for $x \in \Omega$ and $R$ small enough. However due to estimate $(4.1)$ both $M(u, x ; R)$ and $N(u, x ; R)$ as functions of $R$ can be extended to entire functions of exponential growth locally uniformly in $\Omega$.

Theorem 4.5. Let $\varrho>0$ and $\tau \geq 0$. If $u$ is of Laplacian growth $(\varrho, \tau)$ on $\Omega$, then $M(u, x ; R)$ and $N(u, x ; R)$ as functions of $R$ extend holomorphically to entire functions of exponential growth $\left(\varrho, \tau^{\varrho} / \varrho\right)$ locally uniformly in $\Omega$.

Proof. Let $u$ be of Laplacian growth $(\varrho, \tau)$ on $\Omega$. Since $u$ is real analytic, (3.2) holds for any $\stackrel{\circ}{x} \in \Omega$ and $R$ small enough. Set

$$
\widetilde{M}(u, x ; z)=\sum_{k=0}^{\infty} \frac{\Delta^{k} u(x)}{4^{k}\left(\frac{d}{2}+1\right)_{k} k !} z^{2 k} \quad \text { for } x \in \Omega \text { and } z \in \mathbb{C} .
$$

Then $\widetilde{M}$ is a holomorphic extension of $M$. Indeed, applying (4.1) and the inequality $(2 k) ! \leq 4^{k}\left(\frac{d}{2}+1\right)_{k} k$ !, we get for any $K \Subset \Omega, \varepsilon_{1}>0$ and $R<\infty$,

$$
\begin{aligned}
\sup _{x \in K} \sup _{|z| \leq R}|\widetilde{M}(u, x ; z)| & \leq \sup _{x \in K} \sum_{k=0}^{\infty} \frac{\left|\Delta^{k} u(x)\right|}{4^{k}\left(\frac{d}{2}+1\right)_{k} k !} R^{2 k} \\
& \leq C_{K, \varepsilon_{1}} \sum_{k=0}^{\infty} \frac{(2 k) !^{1-1 / \varrho}\left(\tau+\varepsilon_{1}\right)^{2 k}}{4^{k}\left(\frac{d}{2}+1\right)_{k} \cdot k !} R^{2 k} \\
& \leq C_{\varepsilon_{1}} \sum_{k=0}^{\infty} \frac{1}{(2 k) ! 1 / \varrho}\left(\tau+\varepsilon_{1}\right)^{2 k} R^{2 k} .
\end{aligned}
$$

Clearly, the last series converges for any $R$. So $\widetilde{M}(u, x ; R)$ as a function of $R$ is entire. Now fix $\varepsilon>0$ and find $\varepsilon_{1}>0$ such that $e\left(\tau+\varepsilon_{1}\right)^{\varrho} \leq e \tau^{\varrho}+\varepsilon$. Then using $k ! \geq k^{k} e^{-k}$ for $k \in \mathbb{N}_{0}$, we estimate for any $k \in \mathbb{N}_{0}$,

$$
\frac{1}{(2 k) !^{1 / \varrho}}\left(\tau+\varepsilon_{1}\right)^{2 k} \leq\left(\frac{e}{2 k}\right)^{2 k / \varrho}\left(\tau+\varepsilon_{1}\right)^{2 k} \leq\left(\frac{e \varrho \tau / \varrho+\varepsilon}{2 k}\right)^{2 k / \varrho} .
$$

Thus, by Theorem 4.3, $\widetilde{M}(u, x ; R)$ is an entire function of exponential growth $\left(\varrho, \tau^{\varrho} / \varrho\right)$ locally uniformly in $\Omega$.

In the case of $N(u, x ; R)$ the proof goes along the same lines.

Theorem 4.6. Let $\varrho>0, \tau \geq 0$ and $u \in \mathcal{A}(\Omega)$. Assume that $M(u, x ; R)$ (resp. $N(u, x ; R)$ ) defined for $x \in \Omega$ and $0 \leq R<\operatorname{dist}(x, \partial \Omega)$ extends holomorphically to an entire function $\widetilde{M}(u, x ; z)(\operatorname{resp} . \widetilde{N}(u, x ; z))$ of exponential growth $(\varrho, \tau)$ locally uniformly in $\Omega$. Then $u$ is of Laplacian growth $\left(\varrho,(\tau \varrho)^{1 / \varrho}\right)$ on $\Omega$.

Proof. Clearly, for any $x \in \Omega$ an extension $\widetilde{M}$ of $M$ is given by (4.2). Fix $K \Subset \Omega$ and $\varepsilon>0$. Choose $0<\varepsilon_{3}<\varepsilon_{2}<\varepsilon_{1}<\varepsilon$ satisfying

$$
\begin{aligned}
& \left(1+\varepsilon_{3}\right)^{3 / 2-1 / \varrho}\left((\varrho \tau)^{1 / \varrho}+\varepsilon_{1}\right) \leq(\varrho \tau)^{1 / \varrho}+\varepsilon, \\
& \left(\varrho \tau+\varepsilon_{2}\right)^{1 / \varrho} \leq(\varrho \tau)^{1 / \varrho}+\varepsilon_{1},
\end{aligned}
$$




$$
\frac{1+\varepsilon_{3}}{e} \leq \frac{\varrho \tau+\varepsilon_{2}}{e \varrho \tau+\varepsilon_{2}} .
$$

(To show the existence of $\varepsilon_{2}>0$ satisfying (4.4) set $f\left(\varepsilon_{1}\right)=\left((\varrho \tau)^{1 / \varrho}+\varepsilon_{1}\right)^{\varrho}$. Then $f(0)=\varrho \tau$ and $f^{\prime}\left(\varepsilon_{1}\right)=\varrho\left((\varrho \tau)^{1 / \varrho}+\varepsilon_{1}\right)^{\varrho-1}>0$ for $\varepsilon_{1}>0$.) By Theorem 4.3 the assumption that $\widetilde{M}$ is an entire function of exponential growth $(\varrho, \tau)$ uniformly on $K$ implies that we can find $C_{\varepsilon_{2}}$ such that

$$
\sup _{x \in K} \frac{\left|\Delta^{k} u(x)\right|}{4^{k}\left(\frac{d}{2}+1\right)_{k} k !} \leq C_{\varepsilon_{2}}\left(\frac{e \varrho \tau+\varepsilon_{2}}{2 k}\right)^{2 k / \varrho} \quad \text { for } k \in \mathbb{N} .
$$

We can also find $C_{\varepsilon_{3}}$ such that

$$
\left(\frac{d}{2}+1\right)_{k} \leq C_{\varepsilon_{3}}\left(1+\varepsilon_{3}\right)^{k} k ! \quad \text { for } k \in \mathbb{N}_{0}
$$

and (by the Stirling formula)

$$
(k / e)^{k} \leq k ! \leq C_{\varepsilon_{3}}(k / e)^{k}\left(1+\varepsilon_{3}\right)^{k} \quad \text { for } k \in \mathbb{N}_{0} .
$$

Hence for $k \in \mathbb{N}$ we get

$$
\begin{aligned}
\sup _{x \in K}\left|\Delta^{k} u(x)\right| & \leq 4^{k}\left(\frac{d}{2}+1\right)_{k} k ! \cdot C_{\varepsilon_{2}}\left(\frac{e \varrho \tau+\varepsilon_{2}}{2 k}\right)^{2 k / \varrho} \\
& \leq 4^{k} C_{\varepsilon_{2}} C_{\varepsilon_{3}}\left(1+\varepsilon_{3}\right)^{k}(k !)^{2}\left(\frac{e \varrho \tau+\varepsilon_{2}}{2 k}\right)^{2 k / \varrho} .
\end{aligned}
$$

Now by (4.7) and (4.5),

$$
(k !)^{2 / \varrho} \leq C_{\varepsilon_{3}}^{2 / \varrho}\left(\frac{k\left(1+\varepsilon_{3}\right)}{e}\right)^{2 k / \varrho} \leq C_{\varepsilon_{3}}^{2 / \varrho}\left(\frac{k\left(\varrho \tau+\varepsilon_{2}\right)}{e \varrho \tau+\varepsilon_{2}}\right)^{2 k / \varrho} .
$$

So

$$
(k !)^{2 / \varrho} \cdot\left(\frac{e \varrho \tau+\varepsilon_{2}}{2 k}\right)^{2 k / \varrho} \leq C_{\varepsilon_{3}}^{2 / \varrho}\left(\frac{\varrho \tau+\varepsilon_{2}}{2}\right)^{2 k / \varrho} .
$$

Thus by (4.8), (4.4), (4.7) and (4.3), we derive

$$
\begin{aligned}
\sup _{x \in K}\left|\Delta^{k} u(x)\right| & \leq 4^{k} C_{\varepsilon_{2}} C_{\varepsilon_{3}}\left(1+\varepsilon_{3}\right)^{k}(k !)^{2-2 / \varrho} C_{\varepsilon_{3}}^{2 / \varrho}\left(\frac{\varrho \tau+\varepsilon_{2}}{2}\right)^{2 k / \varrho} \\
& \leq C_{\varepsilon_{2}} C_{\varepsilon_{3}}^{1+2 / \varrho}\left(1+\varepsilon_{3}\right)^{k} 4^{k(1-1 / \varrho)}(k !)^{2-2 / \varrho}\left((\varrho \tau)^{1 / \varrho}+\varepsilon_{1}\right)^{2 k} \\
& \leq C_{\varepsilon_{2}} C_{\varepsilon_{3}}^{3}\left(1+\varepsilon_{3}\right)^{k(3-2 / \varrho)}(2 k) !^{1-1 / \varrho}\left((\varrho \tau)^{1 / \varrho}+\varepsilon_{1}\right)^{2 k} \\
& \leq C_{\varepsilon}(2 k) !^{1-1 / \varrho}\left((\varrho \tau)^{1 / \varrho}+\varepsilon\right)^{2 k} .
\end{aligned}
$$

Since $K \Subset \Omega$ was arbitrary, $u$ is of Laplacian growth $\left(\varrho,(\varrho \tau)^{1 / \varrho}\right)$ on $\Omega$.

In the case of $N$ the proof goes along the same lines. 
5. Convergent solutions of the heat equation. Here we consider the initial value problem for the heat equation

$$
\left\{\begin{array}{l}
\partial_{t} u-\Delta_{x} u=0, \\
\left.u\right|_{t=0}=u_{0}
\end{array}\right.
$$

where $u_{0} \in \mathcal{A}(\Omega), \Omega \subset \mathbb{R}^{d}$. Clearly, the unique formal power series solution of $(5.1)$ is given by

$$
\widehat{u}(t, x)=\sum_{k=0}^{\infty} \frac{\Delta^{k} u_{0}(x)}{k !} t^{k} .
$$

We ask when the solution $u$ is an analytic function of the time variable at $t=0$. In the dimension $d=1$ the problem was solved by Kowalevskaya [9]. She proved that the solution $u$ is analytic in time if and only if the initial data $u_{0}$ can be holomorphically extended to an entire function of exponential order 2. In the multidimensional case the solution of the problem was given by Aronszajn et al. [1] in terms of the growth of iterates of the Laplacian of the initial data.

Theorem 5.1. Let $0<T \leq \infty$. If the formal power series solution (5.2) of the initial value problem $(5.1)$ is convergent for $|t|<T$ locally uniformly in $\Omega$, then $M\left(u_{0}, x ; R\right)$ and $N\left(u_{0}, x ; R\right)$ extend holomorphically to entire functions of exponential growth $(2,1 /(4 T))$ locally uniformly in $\Omega$. Conversely, if $M\left(u_{0}, x ; R\right)$ or $N\left(u_{0}, x ; R\right)$ can be holomorphically extended to entire functions of exponential growth $(2,1 /(4 T))$ locally uniformly in $\Omega$, then the solution (5.2) of (5.1) is convergent for $|t|<T$ locally uniformly in $\Omega$.

Proof. Assume that $\widehat{u}(t, x)$ is convergent for $|t|<T$ locally uniformly in $\Omega$. Then for any compact set $K \Subset \Omega$ and $\varepsilon>0$ there exists $C_{\varepsilon}=C(K, \varepsilon)<\infty$ such that

$$
\sup _{x \in K}\left|\Delta^{k} u_{0}(x)\right| \leq C_{\varepsilon}\left(\frac{1}{T}+\varepsilon\right)^{k} \cdot k ! \quad \text { for } k \in \mathbb{N}_{0} .
$$

So for any $k \in \mathbb{N}_{0}$, we have

$$
\begin{aligned}
\sup _{x \in K}\left|\Delta^{k} u_{0}(x)\right| & \leq C_{\varepsilon}\left(\frac{1}{T}+\varepsilon\right)^{k} \cdot k ! \leq C_{\varepsilon}\left(\frac{1}{T}+\varepsilon\right)^{k}\left(\frac{1}{2}+\varepsilon\right)^{k} \cdot(2 k) !^{1 / 2} \\
& \leq C_{\varepsilon}\left((2 T)^{-1 / 2}+\varepsilon\right)^{2 k} \cdot(2 k) !^{1 / 2}
\end{aligned}
$$

Hence, $u_{0}$ is of Laplacian growth $(2,1 / \sqrt{2 T})$ on $\Omega$ and by Theorem 4.5, $M\left(u_{0}, x ; R\right)$ and $N\left(u_{0}, x ; R\right)$ extend holomorphically to entire functions of exponential growth $(2,1 /(4 T))$ locally uniformly in $\Omega$.

Conversely, suppose that $M\left(u_{0}, x ; R\right)$ or $N\left(u_{0}, x ; R\right)$ can be holomorphically extended to entire functions of exponential growth $(2,1 /(4 T))$ locally uniformly in $\Omega$. Then by Theorem 4.6, $u_{0}$ is of Laplacian growth $(2,1 / \sqrt{2 T})$ on $\Omega$. Fix $K \Subset \Omega$ and $|t|<T$. Then for $\varepsilon>0$ sufficiently small, we get 


$$
\begin{aligned}
\sup _{x \in K} \sum_{k=0}^{\infty} \frac{\left|\Delta^{k} u_{0}(x)\right|}{k !}|t|^{k} & \leq C_{\varepsilon} \sum_{k=0}^{\infty} \frac{(1 / \sqrt{2 T}+\varepsilon)^{2 k} \cdot(2 k !)^{1 / 2}|t|^{k}}{k !} \\
& \leq C_{\varepsilon} \sum_{k=0}^{\infty}\left(\frac{1}{2 T}+\varepsilon\right)^{k}(2+\varepsilon)^{k}|t|^{k} \\
& \leq C_{\varepsilon} \sum_{k=0}^{\infty}\left[\left(\frac{1}{T}+\varepsilon\right)|t|\right]^{k}<\infty .
\end{aligned}
$$

Since $K \Subset \Omega$ was arbitrary, $\widehat{u}(t, x)$ is convergent for $|t|<T$ locally uniformly in $\Omega$.

Remark 5.2. The problem of Borel summability of formal solutions of the heat equation was studied by Michalik [11]. From his main result [11, Theorem 1] one can derive a characterization of analytic solutions of (5.1) similar to that in Theorem 5.1.

Open Access. This article is distributed under the terms of the Creative Commons Attribution Noncommercial License which permits any noncommercial use, distribution, and reproduction in any medium, provided the original author(s) and source are credited.

\section{References}

[1] N. Aronszajn, T. M. Creese, And L. J. Lipkin, Polyharmonic Functions, Clarendon Press, Oxford, 1983.

[2] R. P. BoAs JR, Entire Functions, Academic Press, New York, 1954.

[3] B. Bojanov, An extension of the Pizzetti formula for polyharmonic functions, Acta Math. Hungar. 91 (2001), 99-113.

[4] J. H. Bramble and L. E. Payne, Mean value theorems for polyharmonic functions, Amer. Math. Monthly 73 (1966), 124-127.

[5] G. M. Fichtenholz, Differential- und Integralrechnung, vol. III, Hochschulbücher für Mathematik 63, Johann Ambrosius Barth Verlag GmbH, Leipzig, 1992.

[6] A. Friedman, Mean-values and polyharmonic polynomials, Michigan Math. J. 4 (1957), 67-74.

[7] M. Ghermanesco, Sur les moyennes successives des fonctions, Bull. Soc. Math. France 62 (1934), 245-264.

[8] M. Ghermanescu, Sur les moyennes successives des fonctions, Math. Ann. 119 (1943), 288-320.

[9] S. Kowalevski, Zur Theorie der partiellen Differentialgleichungen, J. Reine Angew. Math. 80 (1875), 1-32.

[10] G. Łysik, On the mean-value property for polyharmonic functions, Acta Math. Hungar. 133 (2011), 133-139.

[11] S. Michalik, Summability of divergent solutions of the $n$-dimensional heat equation, J. Differential Equations 229 (2006), 353-366. 
[12] R. Narasimhan, Analysis on Real and Complex Variables, Masson, Paris and North-Holland, Amsterdam, 1968.

[13] M. Nicolesco, Les Fonctions Polyharmoniques, Hermann, Paris, 1936.

[14] P. Pizzetti, Sulla media dei valori che una funzione dei punti dello spazio assume alla superficie di una sfera, Rendiconti Lincei, serie V, 18 (1909), $182-185$.

[15] P. Pizzetti, Sul significato geometrico del secondo parametro differenziale di una funzione sopra una superficie qualunque, Rendiconti Lincei, serie V, 18 (1909), 309-316.

[16] J. H. Sampson, On Pizzetti's formula, Boll. Un. Mat. Ital., serie 6, A 2, (1983), 99-102.

[17] F. Sbrana, Sopra una proprietà caratteristica delle funzioni poliarmoniche e delle soluzioni dell'equazione delle membrane vibranti, Rendiconti Lincei, serie VI, 1 (1925), 369-371.

[18] L. Zalcman, Mean values and differential equations, Israel J. Math. 14 (1973), 339-352.

GRZEGORZ EYSIK

Institute of Mathematics,

Polish Academy of Sciences, Śniadeckich 8,

00-956 Warsaw 10,

Poland

and

Jan Kochanowski University,

Kielce, Poland

e-mail: lysik@impan.pl

Received: 15 March 2011

Revised: 21 October 2011 